Prepared For SUbMission to JINST

$15^{\text {TH }}$ Topical Seminar on Innovative Particle and Radiation Detectors (IPRD19)

14-17 OCTOBER 2019

SiEnA, ITALY

\title{
First results on the performance of the PADME electromagnetic calorimeter
}

\author{
G. Piperno, ${ }^{a, b, 1}$ on behalf of PADME collaboration \\ ${ }^{a}$ Dipartimento di Fisica, Sapienza - Università di Roma, Roma I-00185, Italy \\ ${ }^{b}$ INFN - Sezione di Roma, Roma I-00185, Italy \\ E-mail: gabriele.piperno@roma1.infn.it
}

\begin{abstract}
The PADME experiment, hosted at the Laboratori Nazionali di Frascati in Italy, is dedicated to the dark photon search. It looks for the reaction $e^{+} e^{-} \rightarrow A^{\prime} \gamma$, where $A^{\prime}$ indicates the dark photon. The $550 \mathrm{MeV}$ positrons beam that impinges on electrons, coming from an active diamond target, allows to scan $A^{\prime}$ masses up to $23.7 \mathrm{MeV}$. In this context, the segmented electromagnetic calorimeter plays a fundamental role, since it measures the final photon fourmomentum. It consists of $616 \mathrm{BGO}$ crystals displaced in a cylindrical shape of $\approx 29 \mathrm{~cm}$ radius with a central square hole of 5 crystals side, to let Bremsstrahlung radiation pass, to limit the calorimeter trigger rate. Each crystal is read by a HZC XP1911 type B photomultipliers and their signal is digitised by means of a CAEN V1742 board, taking 1024 samples at $1 \mathrm{GS} / \mathrm{s}$. Here we report in detail the solutions adopted for the calorimeter together with the results obtained in tests performed on a small prototype and on single scintillating units.
\end{abstract}

Keywords: Calorimeters, Scintillators, Gamma detectors, Detector design and construction technologies and materials

${ }^{1}$ Corresponding author. 


\section{Contents}

1 Introduction 1

2 The PADME experiment 2

3 The PADME electromagnetic calorimeter 3

3.1 Scintillating units calibration with ${ }^{22} \mathrm{Na} \quad 5$

$\begin{array}{ll}3.2 & \text { Cosmic rays in the electromagnetic calorimeter } \\ 3.3 & \text { Beam }\end{array}$

3.3 Beam test on a calorimeter prototype 6

4 Conclusions $\quad 6$

\section{Introduction}

As a result of many cosmological and astronomical observations we know that only $15 \%$ of the mass of the Universe is made of Standard Model (SM) matter. The largest part seems to consist of an invisible form of matter, called Dark Matter (DM) [1]. Despite all these results, DM nature is still unknown.

A possible solution to the problem of its elusiveness is that it lives in a separate sector, named Dark Sector, and that it interacts with the SM only by means of portals. The simplest connection between our world and the Dark Sector can be built introducing a new U(1) symmetry, which adds a new vector boson, the Dark Photon (DP) $A^{\prime}[2,3]$. Even if the SM particles are neutral under this symmetry, the DP can interact with them via a kinetic mixing with the ordinary photon. Consequently, particles will acquire an effective charge $\varepsilon q$, where $\varepsilon$ is the $A^{\prime}$ coupling constant and $q$ is the particle electric charge.

A strong point of this model is that it is very predictive, being completely described when the constant $\varepsilon$ and the mass $m_{A^{\prime}}$ are defined.

Generally the DP search is divided into two classes, depending on the decay channel type: visible or invisible. The first case occurs when there are no DM particles with mass $\leq m_{A^{\prime}} / 2$ and the $A^{\prime}$ boson is forced to decay into SM particles. On the contrary, when DM particles with mass $\leq m_{A^{\prime}} / 2$ exist, the $A^{\prime}$ boson will decay mostly into this kind of particles and the SM channels are then suppressed by a factor $\varepsilon^{2}$.

The DP can also solve other existing tensions of the SM. Depending on the model, it could explain, partially or completely, the discrepancy between the expected value and the measured one of the muon anomalous magnetic moment $(g-2)_{\mu}[4]$ and the two recent observations known as the Beryllium-8 and Helium-4 anomaly [5, 6].

In refs. [7, 8] a more complete panorama of the DP physics and current research approaches are presented. 


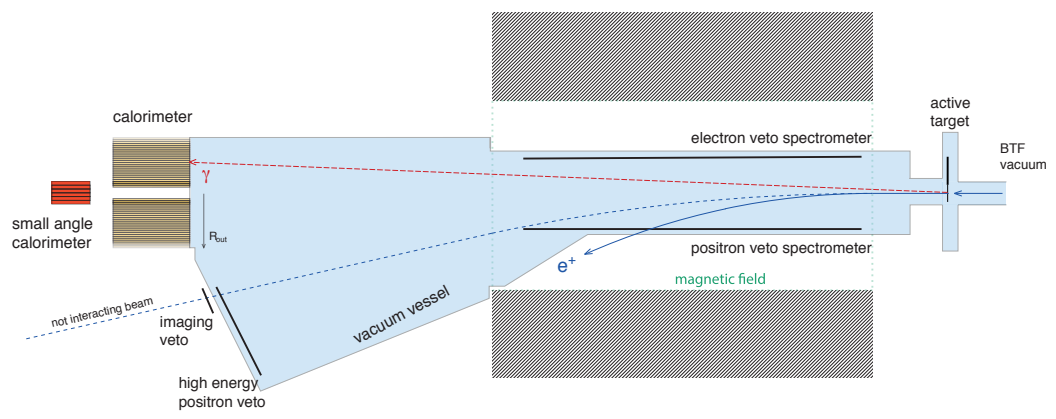

Figure 1. PADME detector scheme, with positron beam arriving from the right: diamond active target, magnetic dipole with electron/positron vetoes inside and high energy positron veto and TimePix 3 array, both close to the beam exit; at $3.45 \mathrm{~m}$ from the target are placed the electromagnetic calorimeter and, $50 \mathrm{~cm}$ behind it, the small angle calorimeter.

\section{The PADME experiment}

The main aim of the Positron Annihilation into Dark Matter Experiment (PADME), hosted at the Laboratori Nazionali di Frascati, is the search for an invisibly decaying DP. Placed in the laboratories Beam Test Facility [9], it exploits the LINAC to accelerate positrons that then annihilate with electrons coming from a target, to look for the reaction:

$$
e^{+} e^{-} \rightarrow A^{\prime} \gamma
$$

Positrons are accelerated up to $550 \mathrm{MeV}$, while the electrons are at rest in a diamond active target. If produced, a long lived or invisibly decaying $A^{\prime}$ can't be observed by the experiment. On the contrary, the $\gamma$ four-momentum can be measured by means of a granular electromagnetic calorimeter. Consequently, the $A^{\prime}$ boson can be observed as missing mass that, knowing the initial conditions $\left(\vec{P}_{e^{-}}=\overrightarrow{0} \mathrm{MeV} / \mathrm{c}\right.$ and $\left.\vec{P}_{e^{+}}=(0,0,550) \mathrm{MeV} / \mathrm{c}\right)$, can be measured as (the $\underline{P}$ notation indicates the relativistic four-momentum):

$$
M_{\text {miss }}^{2}=\left(\underline{P}_{e^{-}}+\underline{P}_{e^{+}}-\underline{P}_{\gamma}\right)^{2} .
$$

Requiring only the coupling to leptons, the PADME DP search results to be almost model independent.

Figure 1 represents the scheme of the detector. Moving from the right to the left of the image, it is composed of the following elements:

- The active target consists of a $100 \mu \mathrm{m}$ thick and $2 \times 2 \mathrm{~cm}^{2}$ area polycrystalline diamond, with 19 horizontal and 19 vertical graphitic stripes (only 16 are connected). It returns average information about the beam intensity and position (resolution $\approx 5 \mathrm{~mm}$ ). The material is chosen to reduce the Bremsstrahlung cross section, while the thickness is a balance between threshold (the minimum number of detectable positrons), which decreases with thickness, and the beam multiple scattering, which increases with thickness. 
- The magnetic dipole, a MBP-S dipole $1 \mathrm{~m}$ long with $23 \mathrm{~cm}$ gap and $\approx 0.5 \mathrm{~T}$ field, is placed $20 \mathrm{~cm}$ after the target. Its purpose is to bend beam particles sending them towards the experiment exit, if did not annihilate on the target, or to the charged particle veto system, if they lost part of their energy by Bremsstrahlung.

- The charged particle veto system is the sum of different components: two arrays of plastic scintillating bars inside the magnet gap, one for $e^{+}$(90 cm long), one for $e^{-}$(96 cm long), needed to identify either positrons that lost a large amount of energy for Bremsstrahlung, or the electrons and positrons produced in $e^{+} e^{-} \rightarrow e^{+} e^{-}$interactions. Another array $(36 \mathrm{~cm}$ long), close to the beam exit, is used to detect positrons that lost a small amount of energy for Bremsstrahlung. Each scintillating bar size is $1 \times 1 \times 16 \mathrm{~cm}^{3}$.

- An array of $6 \times 2$ TimePix 3 silicon detectors, for a total size of $8.45 \times 2.82 \mathrm{~cm}^{2}$, is positioned at the beam exit to study bunch divergence and structure, thanks to its spatial (55 $\mu \mathrm{m}$ pitch) and time $(<0.5 \mathrm{~ns})$ resolutions.

- On the opposite side of the experiment from the target there is the Electromagnetic Calorimeter (ECal). It presents a central square opening that allows the Bremsstrahlung radiation to pass and to be detected by a faster calorimeter. Additional information on the ECal is given in the next section.

- The small angle calorimeter, placed $50 \mathrm{~cm}$ behind the $\mathrm{ECal}$, has a square shape and is made of $253 \times 3 \times 14 \mathrm{~cm}^{3}$ lead difluoride $\left(\mathrm{PbF}_{2}\right)$ crystals. The readout signal is Cherenkov light $(\approx 3$ ns duration), which makes the detector able to sustain an event rate up to hundreds of MHz.

A signal event, $e^{+} e^{-} \rightarrow A^{\prime} \gamma$, would appear as a single $\gamma$ in the ECal with no hits in the small angle calorimeter or vetoes and some missing energy, corresponding to the amount taken away by the $A^{\prime}$ boson. Given the beam energy of $550 \mathrm{MeV}$, the maximum $A^{\prime}$ mass that can be explored is 23.7 MeV.

The PADME $90 \%$ C.L. sensitivity to an $A^{\prime}$ boson that decays in the invisible channel is presented in figure 2 . Here the result is given assuming the kinetic mixing model introduced in the previous section and for two different statistics: $10^{13}$ and $4 \cdot 10^{13}$ positrons on target.

\section{The PADME electromagnetic calorimeter}

The ECal, together with the active target, is the most important component of the experiment, given that it measures the four-momentum of the photon in the final state. The chosen material is BGO (bismuth germanate) because of its small radiation length $\left(X_{0}=1.12 \mathrm{~cm}\right)$ and because the PADME collaboration had the opportunity to reshape and reuse crystals from L3 electromagnetic calorimeter endcaps [10]. It is composed of 616 BGO scintillating crystals of dimensions $2.1 \times 2.1 \times 23.0 \mathrm{~cm}^{3}$, displaced in an almost cylindrical shape with a radius of about $29 \mathrm{~cm}$. Given the long decay time of the BGO scintillation light, $300 \mathrm{~ns}$, the ECal is not able to sustain high particle rates. Consequently, it has a central square hole of $5 \times 5$ crystals to let the Bremsstrahlung radiation, very intense at 


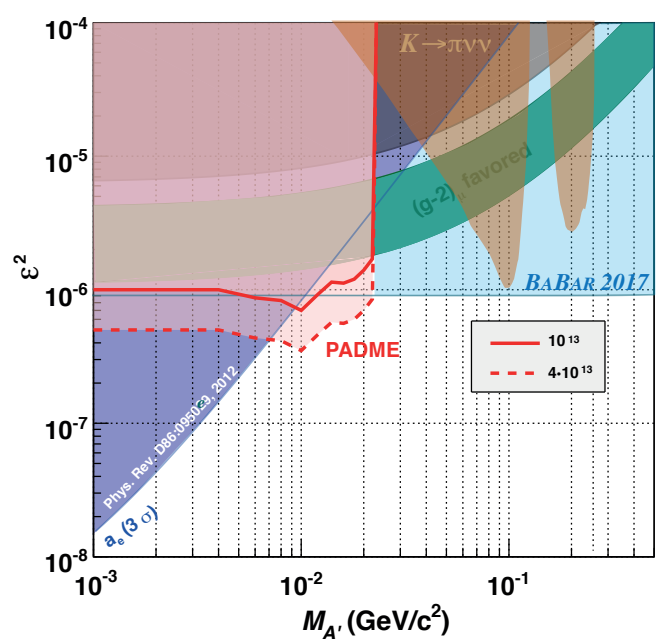

Figure 2. PADME $90 \%$ C.L. sensitivity to a DP that decays into DM particles considering two different amount of positrons on target: $10^{13}$ and $4 \cdot 10^{13}$.

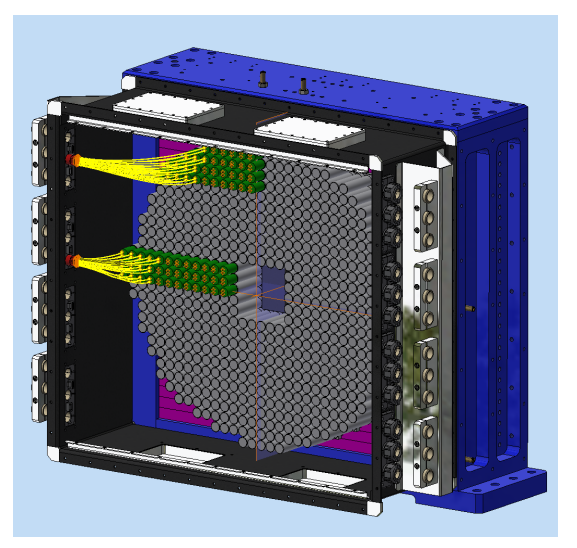

Figure 3. Render of the PADME ECal, with some of the PMTs (orange) and cables (yellow) drawn.

small angles, pass and be observed by the faster small angle calorimeter. With the current distance of $3.45 \mathrm{~m}$ from the active target, the ECal angular coverage is $[15,84] \mathrm{mrad}$.

A photomultiplier tube (PMT) is glued to each BGO crystal to form the so called Scintillating Unit (SU). The selected model is the HZC XP1911 type B, which fits the crystals' square cross section well, thanks to the diameter of $1.9 \mathrm{~cm}$, and the $\mathrm{BGO}$ emission spectrum, that has a maximum at $480 \mathrm{~nm}$, at which wavelength the quantum efficiency of the PMT(s) is 21\% [11]. A render of the calorimeter is shown in figure 3, in which some of the PMTs (in orange) and signal and HV cables (in yellow) can be seen.

The ECal does not have a honeycomb structure in which to insert the various SUs, due to the fact that it would have spoiled the energy resolution of the calorimeter. Therefore one layer of crystals acts as basis for the next layer. To reduce the light crosstalk, $50 \mu \mathrm{m}$ black Tedlar foils are placed between the SUs. These foils are also used to level out any difference in crystal heights by varying the number of layers. Since the start of calorimeter operations in October 2018, 4 SUs have never worked, corresponding to less than the $0.7 \%$ of the total. 


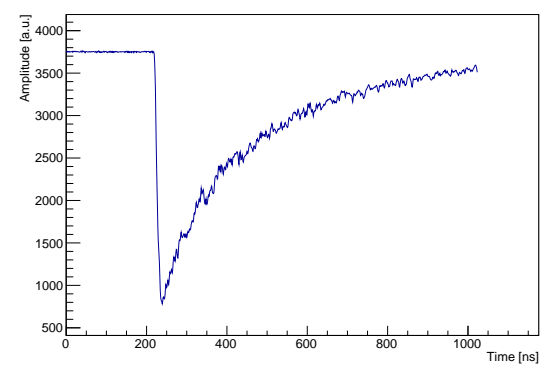

Figure 4. An example of a digitised PADME ECal SU signal.

SUs signals are digitised by means of CAEN V1742 boards. Each board is equipped with 4 DRS4 chips, for a total of 32 channels. The channels dynamic range is $[-1,0] \mathrm{V}$ with a 12-bit precision, while the number of sampled points is 1024 . These boards can acquire data at different sampling frequencies: $1 \mathrm{GS} / \mathrm{s}, 2.5 \mathrm{GS} / \mathrm{s}$ and $5 \mathrm{GS} / \mathrm{s}$. Given the long BGO signals (example in figure 4), the selected one is $1 \mathrm{GS} / \mathrm{s}$.

Temperature variations are extremely important when using BGO, because its light yield varies as $-0.9 \% /{ }^{\circ} \mathrm{C}$. To reduce as much as possible this variability a new air conditioning system was installed in the BTF. It is foreseen that the temperature will remain within $\pm 0.5^{\circ} \mathrm{C}$ the desired value. In addition, PT1000 are glued on crystals to monitor their temperature: 24 SUs have a thermometer on their back, close to the PMT, while other 16 have two sensors glued on the side, at 1/3 and at $2 / 3$ of crystal length, to probe any dependency on the distance from the front face.

\subsection{Scintillating units calibration with ${ }^{22} \mathrm{Na}$}

Before inserting the SUs in the ECal, each of them has been calibrated by means of a ${ }^{22} \mathrm{Na}$ source. The reason for the choice of this isotope is to exploit its back-to-back emission of $2511 \mathrm{keV} \gamma \mathrm{s}$ coming from an $e^{+} / e^{-}$annihilation. The calibration setup consists of a $5 \times 5$ SUs matrix in front of which the source is moved by a couple of step motors. The trigger signal is provided by a $3 \times 3 \times 20 \mathrm{~mm}^{3}$ LYSO crystal read by a SiPM, positioned on the other side of the source with respect to the SU: a BGO window is acquired every time that the LYSO signal exceeds a threshold. In figure 5 a scheme of the setup is presented.

To construct the charge vs HV curve, all the SUs were measured at 10 different voltages in the interval $[1100,1550] \mathrm{V}$, in steps of $50 \mathrm{~V}$, taking about 6000 events per voltage. For $135 \mathrm{SUs}$, this measurement was repeated twice, to evaluate the stability of the obtained results. Each set of data is fitted with the function $Q=A \cdot V^{s}$ ( $Q$ is the charge, $V$ is the HV and $A$ and $s$ are the fit parameters), that allows to get the HV value for the desired gain. An example for a single SU, with the repeated measurement and fit on both groups of data, is given in figure 6 .

The HV distribution obtained for all the ECal SUs, when requiring a gain of $15.3 \mathrm{pC} / \mathrm{MeV}$, is shown in figure 7. It presents an average value of $1186 \mathrm{~V}$ with a standard deviation of $53 \mathrm{~V}$, meaning that at this gain the typical voltage is well below the maximum safe value of $1700 \mathrm{~V}$ and that there is a low variability between the units. This gain is chosen because it is at the centre of the linearity range and leaves room for signals up to $1 \mathrm{GeV}$ to be fully contained in the DAQ without saturating. This is necessary due to the possibility of having events with more than one photon in 
the same SU. In these cases the pulse amplitude from each photon may sum to a value larger than the amplitude given by a single particle of the maximum energy.

\subsection{Cosmic rays in the electromagnetic calorimeter}

To study and improve the ECal performances, also Cosmic Rays (CRs) are exploited. In figure 8 the CRs trigger structure and logic are shown. It consists of two scintillating paddles, one above and one below the ECal, each one read by two PMTs, one per each side of the paddle. PMTs on the same paddle are in logic AND. The logic OR of these two ANDs gives the trigger signal. The OR is needed to maximise the trigger rate and to acquire also cosmics that cross the ECal diagonally, which pass through a single paddle.

In figure 9 an example of a CR passing through the ECal is shown. The color scale, as the number in the box, indicates the charge integral of the SU pulse in $\mathrm{pC}$.

Using CRs it is possible to evaluate the gain homogeneity between the units and improve the ECal energy resolution [13]. The selection of CRs vertically passing through a crystal ensures a smaller spread of the crossing path and, hence, of the released energy. Starting from this and knowing that the average amount of released energy is always the same, it is possible to equalise the charge spectra of the SUs.

Contemporaneously, with the vertical CRs selection, it is also possible to study the ECal channels efficiency by checking how many times a CR is seen by a unit when it is seen by the SU above and the SU below [13].

\subsection{Beam test on a calorimeter prototype}

A $5 \times 5$ matrix of pre-production SUs was measured in a test beam at the BTF [14]. The results obtained show an energy resolution compatible with the one from the L3 experiment [10]. In fact the fit to the relative energy resolution, with the function $\frac{\sigma(E)}{E}=\frac{a}{\sqrt{E[\mathrm{GeV}]}} \oplus \frac{b}{E[\mathrm{GeV}]} \oplus c$, gives the following parameters: $a=2.0 \%, b=0.003 \%$ and $c=1.1 \%$. Consequently the target energy resolution of $2 \% / \sqrt{E}$ has been achieved.

In the same test beam also the linearity of the charge as a function of the beam energy has been studied and resulted to remain within the $2 \%$ up to $1 \mathrm{GeV}$.

Preliminary studies, using the BTF positron beam directly on the ECal, indicate that it shows an energy resolution similar or better with respect to the one obtained with the prototype [13].

\section{Conclusions}

The PADME experiment main purpose is the search for a dark photon $\left(A^{\prime}\right)$ that decays into dark matter particles, looking for the reaction $e^{+} e^{-} \rightarrow A^{\prime} \gamma$. In this context the electromagnetic calorimeter of the experiment plays a fundamental role, allowing the measurement of the final state photon four-momentum and, hence, the $A^{\prime}$ boson mass, as the reaction missing mass. In this paper we describe the calorimeter with the solutions adopted during its construction aimed to improve its performance, like the scintillating material choice, the presence of black Tedlar to reduce the light crosstalk, the absence of a metal structure for the calorimeter and the usage of PT1000 sensors for temperature monitoring. 


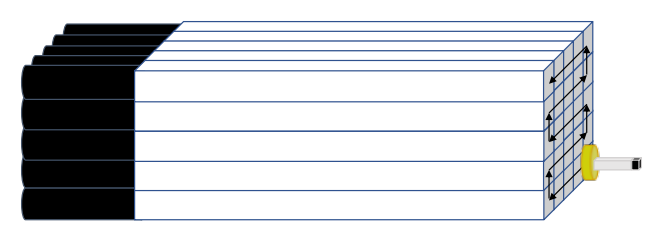

Figure 5. Scheme of the setup for the SUs calibration with the ${ }^{22} \mathrm{Na}$ source. The ECal SUs are on the left, on the right there is the trigger: a LYSO read by a SiPM. The yellow disk between the two, encloses the source.

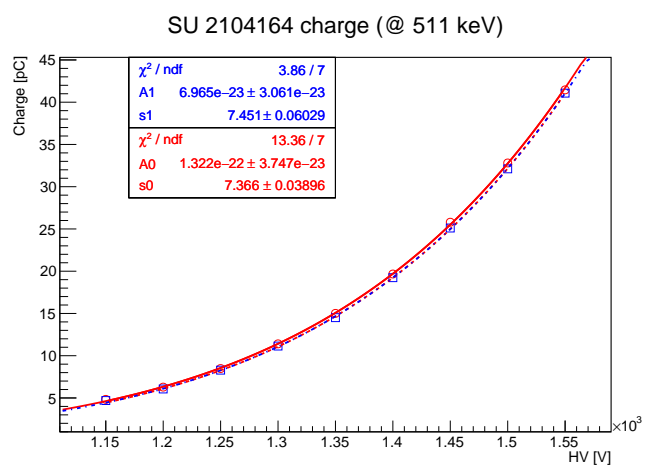

Figure 6. Charge corresponding to a $511 \mathrm{keV}$ photon from the ${ }^{22} \mathrm{Na}$ source as a function of the HV for two measurements performed on the same ECal SU. Only statistical errors are shown.



Figure 7. HV distribution for an equalisation of the ECal SUs at $15.3 \mathrm{pC} / \mathrm{MeV}$.

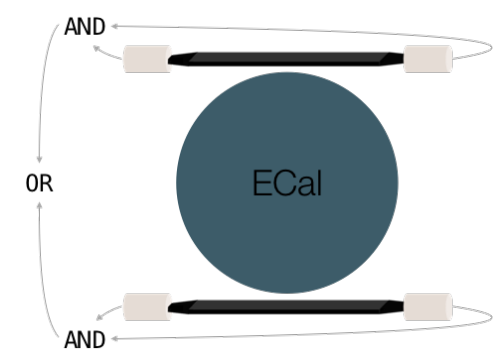

Figure 8. The PADME ECal CRs trigger structure and logic. Two scintillating paddles, one above and one below the ECal, each read by a pair of PMTs. The PMTs on the same paddle are in logic AND. The logic OR of these two ANDs gives the trigger signal. 


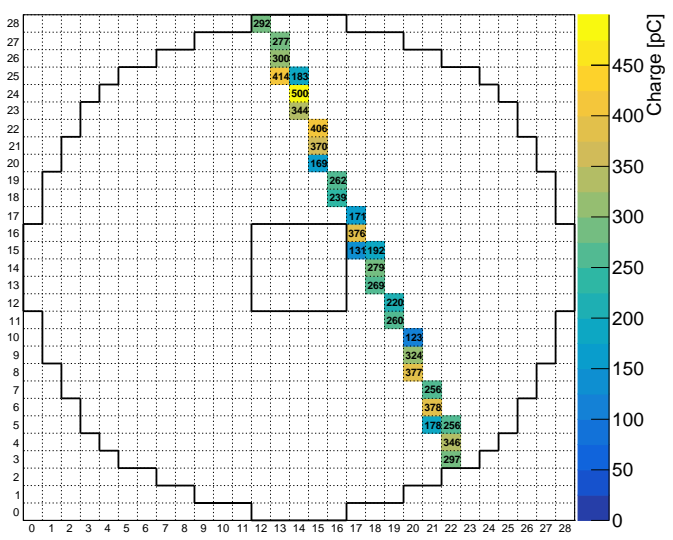

Figure 9. Example of a CR track in the ECal. The colour scale indicates the charge collected by each SU.

In addition the calorimeter cosmic rays trigger is described, together with the possible studies that can be performed by exploiting this information.

As a last argument, the results obtained with a calorimeter prototype are summarised, showing how the observed performance are in line with design ones.

\section{References}

[1] Y. Akrami et al., Planck 2018 results. I. Overview and the cosmological legacy of Planck, arXiv: 1807.06205

[2] P. Galison and A. Manohar, Two Z's or not two Z's?, Phys. Lett. B 136 (1984) 279.

[3] B. Holdom., Two U(1)'s and $\epsilon$ charge shifts, Phys. Lett. B 166 (1986) 196.

[4] M. Pospelov, Secluded U(1) below the weak scale, Phys. Rev. D 80 (2009) 095002.

[5] A. J. Krasznahorkay et al., Observation of Anomalous Internal Pair Creation in ${ }^{8}$ Be : A Possible Indication of a Light, Neutral Boson, Phys. Rev. Lett. 116 (2016) 042501.

[6] A. J. Krasznahorkay et al., New evidence supporting the existence of the hypothetic X17 particle, arXiv: 1910.10459 .

[7] M. Raggi and V. Kozhuharov, Results and perspectives in dark photon physics, Riv. Nuovo Cim. $\mathbf{3 8}$ (2015) 449.

[8] M. Battaglieri et al., US Cosmic Visions: New Ideas in Dark Matter 2017: Community Report, arXiv:1707.04591.

[9] P. Valente et al., Linear Accelerator Test Facility at LNF Conceptual Design Report, INFN-16-04-LNF, arXiv:1603.05651.

[10] B. Adeva et al., The construction of the L3 experiment, Nucl. Instrum. Methods A 289 (1990) 35.

[11] http://www.hzcphotonics.com/

[12] E. Leonardi et al., Development and test of a DRS4-based DAQ system for the PADME experiment at the DA $\Phi N E$ BTF, J. Phys. Conf. Ser. 898 (2017) 032024.

[13] PADME coll., Article in preparation. 
[14] M. Raggi et al., Performance of the PADME Calorimeter prototype at the DA $\Phi N E$ BTF, Nucl. Instrum. Meth. A 862 (2017) 31. 